\title{
Statistical Investigation of Wind Duration Using A Refined Hurricane Track Model
}

\author{
Haifeng Wang ${ }^{\mathrm{a}, *}$, Teng $\mathrm{Wu}^{\mathrm{b}}$ \\ ${ }^{a}$ Department of Civil, Structural and Environmental Engineering, University at Buffalo, Buffalo, \\ New York, United States, hwang48@buffalo.edu \\ ${ }^{b}$ Department of Civil, Structural and Environmental Engineering, University at Buffalo, Buffalo, \\ New York, United States, tengwu@buffalo.edu
}

\begin{abstract}
:
A complete characterization of the atmospheric boundary-layer winds essentially involves speed, direction and duration. While extensive statistical analyses have been carried out to determine wind speed and directionality for appropriate wind design of buildings, there is a lack of similar research effort for wind duration. Recent advances of performance-based wind design methodology allow the controlled nonlinear, inelastic deformation of buildings under strong winds, and hence place a demand on statistical investigation of wind duration. In this study, the wind duration is measured with the over-threshold method (i.e., uniform duration) using a refined hurricane track model. A statistical analysis framework is developed to jointly consider wind duration, speed and direction. A case study is conducted to demonstrate the efficacy of the proposed statistical analysis framework for characterization and quantification of wind duration.
\end{abstract}

Keywords: Wind duration, Performance-based wind design, Refined hurricane track model, Statistical Analysis

\section{INTRODUCTION}

The wind design of building is moving towards performance-based methodology, where it is important to consider duration effects on the strong wind-induced nonlinear, inelastic structural response (ASCE, 2019). The accurate and reliable characterization and quantification of wind duration is a critical step in advancing this consideration. To this end, the statistical investigation of wind duration is carried out in this study. The successful implementation of probabilistic hurricane duration analysis requires a large number of wind data using the Monte Carlo simulation method. This study employs a refined hurricane track model with improved modelling of hurricane movement for wind data preparation. It is noted that by itself the wind duration sheds little light on its effect on structural response. Actually, there is a difficulty in decoupling the wind duration consideration from the wind speed and direction. Accordingly, a statistical analysis framework is developed to jointly consider wind duration, wind speed and wind direction. The efficacy of the proposed statistical analysis framework for characterization and quantification of duration in wind climate consideration is demonstrated through a case study.

\section{METHODOLOGY}

\subsection{Refined hurricane track model}

Vickery et al. (2000) utilized the current-step longitude, latitude, translation speed and direction to get the next-step hurricane location. While the beta effect (controlled by both hurricane location and translation) may be well captured in their simulation, the environmental flow is not considered. To accurately assess the duration of hurricanes, an improved translation simulation scheme with 
consideration of both the environmental flow and beta effect is proposed here:

$u_{i+1}=a_{1}+a_{2} \psi_{i+1}+a_{3} \lambda_{i+1}+a_{4} u_{i}+a_{5} U_{i+1,850}+a_{6} U_{i+1,250}+\varepsilon_{a}$

$v_{i+1}=b_{1}+b_{2} \psi_{i+1}+b_{3} \lambda_{i+1}+b_{4} v_{i}+b_{5} V_{i+1,850}+b_{6} V_{i+1,250}+\varepsilon_{b}$

where $u$ and $v$ are respectively the longitudinal and latitudinal storm translation speeds; subscripts $i$ and $i+1$ are respectively the current and next time steps; $\psi$ and $\lambda$ are respectively the latitude and longitude of hurricane center; $U$ and $V$ are respectively the longitudinal and latitudinal environmental flow speed; the subscripts 850 and 250 respectively represent the heights at 850 - and $250-\mathrm{hPa}$ levels; $a_{1}, a_{2}, \cdots, a_{6}$ and $b_{1}, b_{2}, \cdots, b_{6}$ are coefficients obtained by linear regression; $\varepsilon_{a}$ and $\varepsilon_{b}$ are random error terms generated following Student's $\mathrm{T}$ distribution (Cui and Caracoglia, 2016). The coefficients $a_{1}, a_{2}, \cdots, a_{6}$ and $b_{1}, b_{2}, \cdots, b_{6}$ are estimated for $5^{\circ} \times 5^{\circ}$ grids over the Atlantic Ocean and the Gulf of Mexico.

\subsection{Statistical analysis framework}

A large number of wind data are generated using the refined hurricane track model, as well as the corresponding duration data based on the over-threshold method. To comprehensively investigate the characteristics of wind duration, a statistical analysis framework is proposed. Figure 1 depicts each component of the duration statistical analysis framework used in this study to comprehensively investigate wind duration effects on structural performance.

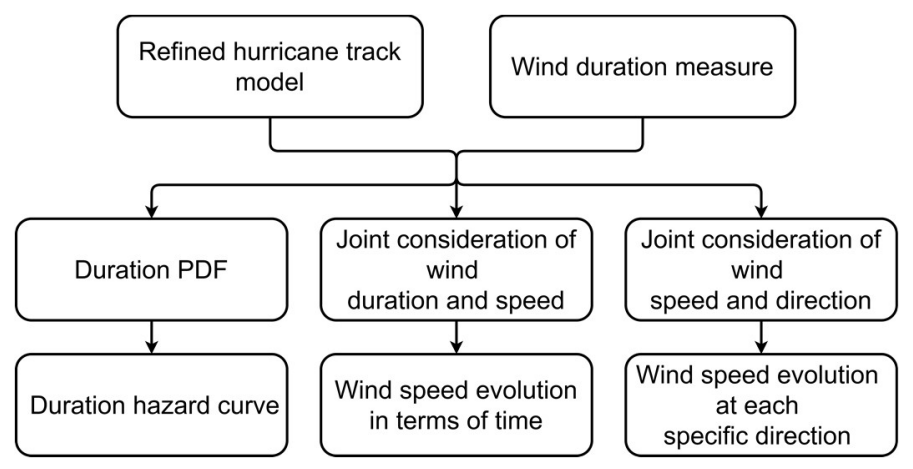

Figure 1. Statistical analysis framework of wind duration

\section{CASE STUDY OF WIND DURATION}

Orlando $\left(28.55^{\circ} \mathrm{N}, 81.38^{\circ} \mathrm{W}\right)$, a hurricane-prone city in Florida, is selected as the site of interest. The wind duration with a threshold wind speed of $46.5 \mathrm{~m} / \mathrm{s}$, corresponding to a mean recurrence interval (MRI) of 50 years (ASCE, 2016), is obtained and analyzed. A total number of 2,160,000 hurricanes are simulated using the refined hurricane track model. Data preprocessing is performed to pre-select the hurricanes that affect the site of interest. There are 2517 hurricanes to be considered.

\subsection{Duration statistical analysis}

Based on the synthesized duration data, their distribution is estimated and presented in Fig. 2. As shown in the figure, the durations are randomly distributed with a mean value of five hours. The fitted Gamma distribution is also presented in Fig. 2. The good match indicates that Gamma 
distribution may be utilized for describing the duration distribution.

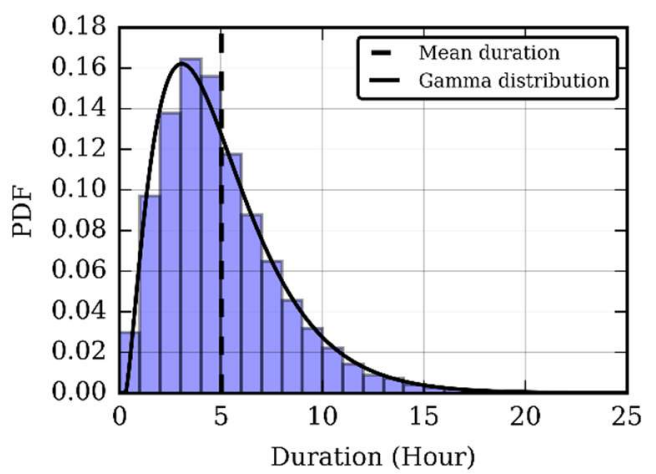

Figure 2. Duration distribution

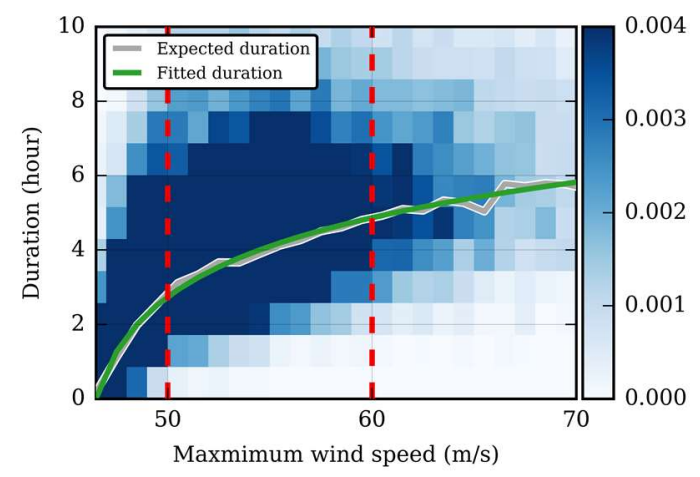

Figure 3. Joint distribution of speed and duration

\subsection{Joint consideration of wind speed and duration}

The duration and the expected wind speed are simultaneously examined here. Figure 3 depicts the joint distribution of wind speed and duration. It is observed that the wind duration is positively related to wind speed. Due to the positive relation between the wind duration and speed, it is important to consider the long durations for extreme hurricane wind events. For the convenience of application, an empirical relation between wind duration and wind speed is fitted as:

$D_{T}=a\left(U-U_{T}\right)^{\frac{1}{2}}$

where $a$ is the shape parameter set as 1.82 here; $U$ is the wind speed; and $U_{T}$ is the threshold wind speed. It is shown that the proposed empirical relation matches well with the duration expectation extracted from simulations. It is noted that the wind duration distributions conditional on wind speeds are also well fitted by Gamma distribution.

\section{REFERENCES}

ASCE, 2016. Minimum Design Loads for Buildings and Other Structures. Structural Engineering Institute of ASCE, Reston, VA.

ASCE, 2019. Prestandard for performance-based wind design. Structural Engineering Institute of ASCE, Reston, VA.

Cui, W. and Caracoglia, L., 2016. Exploring hurricane wind speed along US Atlantic coast in warming climate and effects on predictions of structural damage and intervention costs. Engineering Structures, 122, $209-225$.

Vickery, P. J., Skerlj, P. F., and Twisdale, L. A., 2000. Simulation of hurricane risk in the U.S. using empirical track model. Journal of Structural Engineering, 126(10), 1222-1237. 